\title{
BMJ Open Kids in Action: the protocol of a Youth Participatory Action Research project to promote physical activity and dietary behaviour
}

Manou Anselma, Teatske Altenburg, Mai Chinapaw

To cite: Anselma M, Altenburg T, Chinapaw M. Kids in Action: the protocol of a Youth Participatory Action Research project to promote physical activity and dietary behaviour. BMJ Open 2019;9:e025584. doi:10.1136/ bmjopen-2018-025584

- Prepublication history for this paper is available online. To view these files, please visit the journal online (http://dx.doi org/10.1136/bmjopen-2018025584).

Received 26 July 2018 Revised 7 February 2019 Accepted 5 March 2019
Check for updates

(C) Author(s) (or their employer(s)) 2019. Re-use permitted under CC BY-NC. No commercial re-use. See rights and permissions. Published by BMJ.

Public and Occupational Health, Amsterdam UMC Vrije Universiteit Amsterdam, Amsterdam, The Netherlands

Correspondence to

Manou Anselma;

m.anselma@vumc.nl

\section{ABSTRACT}

Introduction In this study, researchers collaborate with children from a low socioeconomic neighbourhood in Amsterdam in developing, implementing and evaluating interventions targeting their health behaviours. This Youth Participatory Action Research project focuses on the promotion of physical activity and healthy dietary behaviour.

Methods and analysis This study is a controlled trial using participatory methods to develop interventions together with children aged 9-12 years. At four primary schools in a low socioeconomic neighbourhood in Amsterdam, an 'Action Team' is installed: a group of six to eight children who actively participate as co-researchers in developing, implementing and evaluating interventions. An academic researcher facilitates the participatory process. Four control schools, also located in low socioeconomic areas in and around Amsterdam, continue with their regular curriculum and do not participate in the participatory process. For the effect evaluation, physical activity and sedentary behaviour are assessed using accelerometers and self-reporting; dietary behaviour using self-reporting and motor fitness (strength, flexibility, coordination, speed and endurance) using the motor performance fitness test. Effectiveness of the interventions is evaluated by multilevel regression analysis. The process of co-creating interventions and the implemented interventions is continually evaluated during meetings of the Action Teams and with children participating in the interventions. Empowerment of children is evaluated during focus groups. Summaries and transcripts of meetings are coded and analysed to enrich children's findings.

Ethics and dissemination The Medical Ethics Committee of the VU Medical Center approved the study protocol (2016.366).

Trial registration number $\mathrm{TC}=6604$.

\section{INTRODUCTION}

The number of children with overweight or obesity is growing worldwide and this public health problem is high on municipal and governmental agendas. This is no different in the Netherlands, where in 2016, on average, $10.7 \%$ of the children between 8 and 12 years
Strengths and limitations of this study

This study is the first to combine Youth Participatory Action Research (YPAR) with intervention mapping, ensuring that the developed interventions are both evidence-based and matching the interests and needs of the specific target group.

- The study design is a controlled trial, which is unique in YPAR.

- This study is embedded in the community involving a multidisciplinary project group. This aids the sustainability of the interventions.

- This study includes an effect evaluation as well as a process evaluation in which the YPAR process and the empowerment of youth are evaluated.

- Randomisation of schools into the intervention and control group is not possible because of the community approach.

old had overweight/obesity. ${ }^{1}$ In urban areas, such as Amsterdam, the rates exceed the country's average, with prevalence rates of overweight/obesity of $12.8 \%$ among 5-year olds and $20.9 \%$ among 10 -year olds. ${ }^{2}$ Even though the overweight numbers are stabilising, health inequalities still exist ${ }^{3}$ : children with overweight or obesity are not only disproportionately divided geographically, but also across income and ethnic groups. ${ }^{4-6}$ Looking at race/ethnicity, children from minority groups show higher overweight/obesity rates than children from a majority group. ${ }^{48}$ For example, in Amsterdam, $10.4 \%$ of 5-year-old children with a Dutch ethnicity have overweight while this is almost $30 \%$ in 10 -year-old children with a non-Western background. ${ }^{3}$ In relation to income groups, in the Netherlands, in the age category 4-25-year olds, $11.2 \%$ of the highest income group had overweight versus $18.0 \%$ of the lowest income group. ${ }^{9}$ Similarly, in 2017, in Amsterdam, 30.1\% of the 10 -year-old children with a very low socioeconomic status (SES) had overweight versus $9.8 \%$ of the 10 -year-old children with a very 
high SES. ${ }^{3}$ Importantly, children with overweight are at high risk of remaining overweight and are, therefore, also at higher risk for chronic illnesses during childhood and in their adult life. ${ }^{10}$ This is why prevention of overweight in children is a priority for many health organisations, municipalities and ministries. ${ }^{11} 12$

Many interventions have been developed and implemented to prevent childhood obesity, but most show disappointing effects. ${ }^{13} 14$ Pivotal in childhood obesity prevention is improving dietary behaviour, physical activity and sedentary behaviour, ${ }^{15}{ }^{16}$ but this is challenging. ${ }^{17-19}$ Strikingly, the most affected group of children-that is, from families with a low SES and from non-Western backgrounds-is most difficult to reach through interventions, ${ }^{20}$ thereby maintaining or even widening health inequalities. ${ }^{21}{ }^{22}$ One reason why these interventions show low participation and effectiveness in this target group could be because the target group is seldom involved in the development of the interventions. ${ }^{23}$ Involving the target group is essential to connect to their needs and interests, ${ }^{24}$ as this influences the reach and effectiveness of the intervention. Therefore, in the current research project-'Kids in Action'-children from a low SES neighbourhood are engaged as co-researchers, that is, applying Youth Participatory Action Research (YPAR). Children not only co-create interventions to improve their lifestyle and that of their peers and family members, but also collaborate in the implementation and evaluation of these interventions. To structure this process, the systematic intervention mapping (IM) methodology is applied alongside YPAR. Through six iterative steps, the IM protocol guides health promoters in the development of evidence-based interventions to change behaviour. ${ }^{25}{ }^{26}$ Combining IM and YPAR ensures that the co-created interventions are appropriate to the interests and needs of the children, but also build on existing evidence. The application of IM alongside YPAR is a novel approach, which we iteratively shape during this study.

\section{Aims and objectives}

The overall aim of the 'Kids in Action' study (April 2016 to November 2019) is to develop, implement and evaluate interventions that stimulate a healthy lifestyle to reduce health inequalities in children from a low SES neighbourhood in collaboration with the children themselves. This study builds on a participatory needs assessment that was conducted in the same neighbourhood. ${ }^{27}$ From this needs assessment, two main needs were identified: to improve physical activity and a healthy diet. The organised activities should be offered at a low price and at a nearby location, the education concerning a healthy diet should be organised in a fun and practical manner.

The primary objective of this study is to evaluate whether designing interventions in collaboration with children can lead to interventions that are more effective in improving children's physical activity and dietary behaviour.
The secondary objective of this study is to evaluate the process of combining YPAR with IM. This includes evaluating the effects of participating in the YPAR process on the empowerment of children and the judgement of children and other stakeholders of interventions that were co-developed by their peers.

\section{METHODS}

\section{Participatory action research}

PAR aims to 'improve health and reduce health inequities' by working together with the community and consequently empowering the community by getting them to improve their own health. ${ }^{28}$ Throughout the entire process of developing, implementing and evaluating interventions, community members are involved as co-researchers and highly valued as experts of their own lives and experiences. At the same time, the community is empowered and experiences more ownership over their lives and livelihood.

This study specifically works together with children in the PAR process. YPAR engages youth as co-researchers in the research process. In this process, children identify problems in their living environment and become empowered to do something about it. ${ }^{29-32}$ Children learn research skills so they can participate in research and have shared power over the research-making and decision-making processes. ${ }^{30} 3233$

\section{Patient and public involvement}

This study is initiated by academic researchers and a community organisation. The municipality advises on the selection of the intervention neighbourhood, to make sure a neighbourhood is recruited with high health needs that can benefit from the project. As this study is informed by a participatory needs assessment (see the Aims and objectives section), ${ }^{27}$ the objectives and outcome measures of this study are determined in collaboration with children, parents and professionals working with children in the neighbourhood. The design of the study and recruitment procedures are decided by the academic researchers. The conduct of the study, the development of interventions and the dissemination of the results to the study participants and other relevant stakeholders are decided together with the children.

\section{Participants}

The four intervention schools are all situated in one low SES neighbourhood in Amsterdam, where in 2015-2016, over $50 \%$ of the residents had a non-Western background, $27 \%$ of the 10-year olds were overweight/obese and in $2014,31 \%$ of the children under 18 years old grew up in a household defined as low-income. ${ }^{34-36}$ Potential control schools are selected from different neighbourhoods but with similar characteristics regarding overweight/obesity rates, household income and cultural background.

Participants in this study are children from four intervention schools and four control schools in low SES 
neighbourhoods in Amsterdam, the Netherlands. The intervention schools participate in the YPAR process, including implementing and evaluating the developed interventions. The control schools only participate in the measurements for the effect evaluation.

\section{Recruitment}

Following selection of a neighbourhood with high health needs, the intervention schools are contacted by the municipality to inform them about the project and to ask them if they are willing to participate. After the schools agree to participate, the main researcher contacts the schools to give them more information about the project. Control schools in the area of Amsterdam are contacted by the main researcher in a random order via e-mail or telephone until four schools are found that are willing to participate as control schools. Control schools are offered a presentation about the research results after the study is finished.

All children aged 9-12 years (ie, children of the three highest grades in primary school) of the four intervention and four control schools are eligible to participate in the effect measurements of the project. For the YPAR process, children from intervention schools are invited to collaborate with academic researchers in co-researcher groups, named 'Action Teams'. At each of the four intervention schools, one Action Team is formed. For both the effect measurements and the Action Teams, every year new children can participate as the highest grade leaves the school and new children enter the third-highest grade. All children receive an information letter for themselves and for their parents about the measurements and the Action Team. Attached to the information letters for parents is an informed consent letter that at least one of the parents has to sign if they agree to the participation of their child. At all schools, the researcher explains the project in all classes and encourages children to participate, before handing out the information letters. Children who participate in the measurements and/or in the Action Team receive a small gift.

\section{Procedures}

This section describes the five phases of the "Kids in Action' project. See figure 1 for an outline.

\section{Phase 1: creating partnerships}

The first phase consists of creating partnerships with the schools and other stakeholders in the area, such as social workers, organisers of after-school activities and the community centres. Together with these stakeholders, a project group is started that meets every 3 months to discuss running projects in the neighbourhood and how

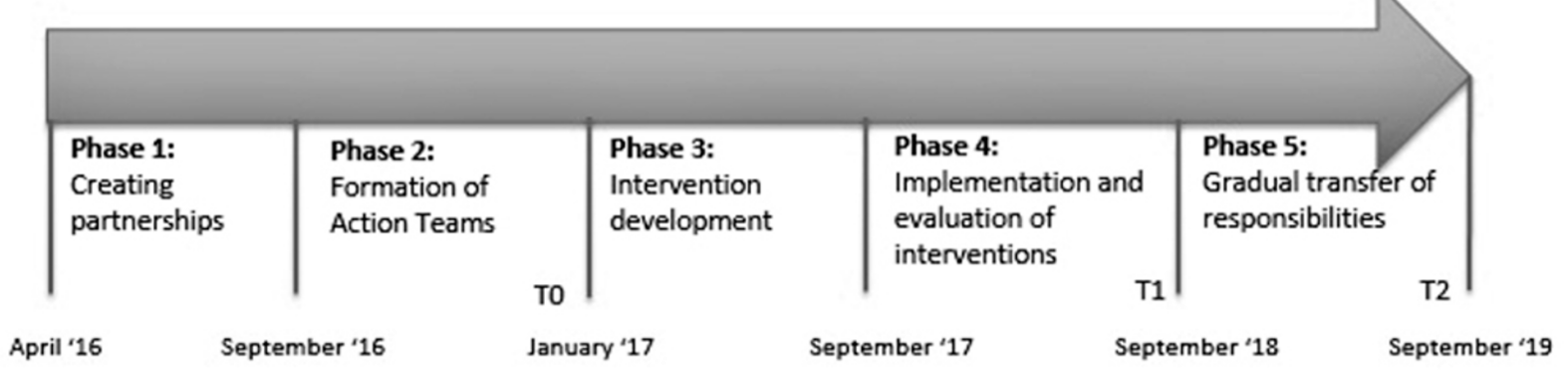

\section{Continuous activities}

Action Team meetings

Capacity building: for children of the Action Teams and Champions

Children involve family-and community members

Process evaluation

Figure 1 Outline of the 'Kids in Action' project. 
partners can collaborate. In this phase, an IM expert group is also formed, to advise on how YPAR and IM should be combined throughout all the phases of the study.

\section{Phase 2: formation of Action Teams}

In the second phase of the project, the Action Teams are formed. Each of the Action Teams consists of six to eight children, an academic researcher and a research assistant. Meetings with the principals of the four intervention schools are planned to decide on recruitment methods for the Action Teams and to schedule the meetings. All interested children aged 9-12 years can sign up for the Action Teams. This approach may lead to bias as only children interested in health may sign up, but limits bias that would occur if teachers select the children for the Action Teams (ie, only the high-performers might be selected). Subsequently, the Action Teams are formed and a general outline of the meetings is developed. In this phase, the baseline effect measurement (T0) is executed.

\section{Phase 3: intervention development}

In the third phase, the meetings of the Action Teams take place. The meetings with the Action Teams are ideally held biweekly during school hours for 1 hour. Despite not all schools agreeing to this in the needs assessment, the researchers try to schedule meetings during school hours to raise the children's motivation for participation. ${ }^{27}$ If the schools do not agree with this, meetings are held weekly for $45 \mathrm{~min}$, followed by a $45 \mathrm{~min}$ sports session. ${ }^{27}$

The first three to four meetings are used to verify the data that was gathered in a participatory needs assessment and to decide on determinants that the interventions need to focus on. ${ }^{27}$ In the rest of the meetings (approximately 10 per year), we develop interventions together with the children targeting children's physical activity and healthy dietary habits. The type of the interventions (eg, environmental changes, organisational changes or educational approaches) is dependent on this collaborative process. Throughout these meetings, capacity building takes place to help the children through the process of intervention development. Children learn, for example, about formulating a research question, different kinds of research methodologies, how to analyse qualitative data, how to translate this data into intervention ideas and practical steps that need to be taken when developing intervention plans. At the end of phase 3, pilots of the first intervention activities are carried out. The Action Teams are also asked to identify 'champions', that is, people who can help them with the development and implementation of the pilots. A champion is a well-known community member, such as a teacher, sports coach or family member. Children discuss who they think is suitable to assist them with a specific intervention and subsequently ask the champions to fulfil this task. The results of this phase (ie, the needs assessment, the intervention ideas and results of the pilots) are discussed with the stakeholders in the project group to make sure the interventions become a joint and sustainable effort.

At the end of the year, a focus group with the Action Teams and their peers is held to discuss the feeling of empowerment that the children of the neighbourhood experience as part of the process evaluation.

\section{Phase 4: implementation and evaluation of interventions}

At the beginning of phase 4, new Action Teams are recruited/formed. Children who were in the Action Teams of the previous year can still participate and are approached first. With the new Action Teams, meetings are planned monthly. Champions are involved and asked to participate in the meetings when appropriate. Together with stakeholders from the project group and the Action Teams, the implementation plans are finalised and subsequently, the interventions are implemented. In order to offer sustainable interventions, we look for partners within the community whose job description aligns with providing the intervention. Depending on the type of intervention, implementers can be dieticians, sports coaches or supermarkets in the community. Once the interventions are implemented, the meetings are used to evaluate the interventions. If the Action Teams feel the interventions are going well, they are encouraged to develop and implement additional intervention activities that focus on other determinants or a different subgroup. ${ }^{33}$ At the end of the year, focus groups are held focusing on empowering children and evaluating interventions. The first effect measurement (T1) is also executed in this phase.

\section{Phase 5: gradual transfer of responsibilities}

In phase 5, responsibilities are gradually transferred to the identified champions. Specific plans are made together with the champions and other stakeholders to continue the interventions and participatory process after this project has ended. The meetings with the (new) Action Teams continue to take place every month, and are used to evaluate and, if necessary, adapt the interventions and discuss new ideas for interventions. The postintervention effect measurement (T2) is executed in this phase. The study ends in November 2019.

\section{Measurements}

\section{Effect evaluation}

The primary outcomes of this study include measures of dietary behaviour (consumption of snacks and sugar-sweetened beverages), physical activity (total Moderate-to-Vigorous Physical Activity (MVPA) time, time spent playing outside and time spent participating in sports), sedentary behaviour (total sedentary time and screen time), self-rated health and physical fitness. Dietary behaviour, physical activity and screen behaviour are measured by self-report. Additionally, physical activity and sedentary behaviour are measured using an accelerometer. Motor fitness is measured using the motor performance (MOPER) fitness test. In the first school year 
(T0), questionnaire and accelerometer data are gathered in the period of September-October 2016 and the fitness test take place in March-April 2017. T1 and T2 take place in March-May 2018 and 2019.

\section{Questionnaire}

A questionnaire is developed containing questions on: the number of small (eg, crisps, nuts and chocolate) and large (eg, hamburger, fries and pizza) snacks children eat; the number of sugar-sweetened beverages they drink; their sports and outdoor play participation; their attitude towards sports and outdoor play; their screen behaviour and their self-rated health. The questionnaire is based on validated items from the ENERGY-child questionnaire, ${ }^{37}$ the DOiT questionnaire ${ }^{38}$ and the EuroQol ${ }^{39}$ Table 1 presents the questionnaire items, and the validity and reliability of the original items.

The children fill in the questionnaire during school hours, in the presence of a researcher who explains the procedure of completing the questionnaire before handing out the questionnaires. The children are requested to go through the questionnaire section by section, with the researcher giving a short explanation about each section before the participants fill in that specific section. In this way, examples can be given, for example, by showing different sizes of soda cans, and all participants finish at the same time. The questionnaire takes approximately $40 \mathrm{~min}$ to complete.

Data entry of the multiple-choice questions is done through digital scanning and transferred into SPSS V.22 by an independent organisation. Qualitative data are manually entered in SPSS.

\section{Accelerometer}

Physical activity and sedentary behaviour are objectively assessed by ActiGraph GT3X+ accelerometers. The children receive instructions and the accelerometers from an academic researcher after filling in the questionnaire. Children are asked to wear the small and light-weight $(4.6 \times 3.3 \times 1.5 \mathrm{~cm} ; 19 \mathrm{~g})$ accelerometer on the right hip for 8 consecutive days during all waking hours except for water-based activities.

The children receive the accelerometer after completing the questionnaire. The children also receive a diary in which the instructions are summarised and they can write down when and why they did not wear the accelerometer, if applicable. Additionally, they are asked to write down the time they went to bed. All children who participate in the questionnaire and accelerometer measurements receive a small present after returning the accelerometer. Additionally, at each school, there is one prize for a participant who wore the accelerometer properly (7 days, at least 10 hours) and recorded their data correctly in their diary.

Data are downloaded from the accelerometers into the ActiLife program in $15 \mathrm{~s}$ epochs. Accelerometer data are analysed using a customised software programme developed in R. We select a cut point of 100 counts per minute $(\mathrm{cpm})$ for sedentary behaviour ${ }^{4041}$ and a cut point of $3000 \mathrm{cpm}$ for MVPA. ${ }^{42}$ Non-wear time is defined as a period of $\geq 60 \mathrm{~min}$ of consecutive zeros. ${ }^{43}$ For inclusion in the data analysis, each participant needs a minimum of 6 days with at least 8 valid hours, including at least 1 weekend day. ${ }^{43}$ Data are analysed on total time spent in MVPA and sedentary, and time in bouts spent in MVPA and sedentary.

\section{Motor performance}

Children's motor fitness is measured using the MOPER test. The MOPER tests speed, flexibility, endurance, coordination and strength by means of eight tests. ${ }^{44}$ For practical reasons, the arm pull and 12-min endurance test have been replaced, leading to the following tests: (1) hang as long as possible on a horizontal bar with flexed arms; (2) jump as high as possible from a standing position; (3) run $10 \times 5 \mathrm{~m}$ as fast as possible; (4) reach as far as possible from a sitting position; (5) hand grip strength measured using a dynamometer ${ }^{45} 46$ (instead of arm pull); (6) lie on their back and lift their extended legs 10 times as fast as possible; (7) tap two plates, which are $75 \mathrm{~cm}$ apart, with the preferred hand 50 times as fast as possible and (8) shuttle run test ${ }^{47} 48$ (instead of 12-min endurance test). Children can do tests number one and eight once. Tests two and five are executed twice, but when the difference between one and two is more than $10 \%$, a third try is performed. The best score is used. The other tests are performed twice and the best score is used. The first seven activities of the MOPER test are executed during one physical education (PE) class by the $\mathrm{PE}$ teacher together with five or six research assistants. The $\mathrm{PE}$ teacher conducts the shuttle run test in a separate PE class. All research assistants and PE teachers are trained by an academic researcher on how the tests should be executed. At the end of the study, or when children from the highest grade leave the school, the PE teacher anonymously shares the results of the test. Parents receive an information letter with a passive consent form, which should be signed by at least one of the parents if they object to anonymously sharing the fitness test results of their child with the researchers.

\section{Process evaluation}

The process evaluation includes the description of the process of co-creating interventions, combining IM and YPAR, and empowerment. The PAR process is continually evaluated in the Action Team meetings, and meetings are optimised in accordance with the evaluation. ${ }^{28}$ The academic researcher and research assistant, who are part of the Action Teams, evaluate after every meeting, using a reflection form consisting of a summary of the meeting, what the setting was like, the group process and a personal reflection. ${ }^{49-51}$

The interventions are developed by combining the YPAR and IM methodologies in an iterative process and are continuously evaluated during the meetings of the Action Teams and with the children participating in the 
Table 1 Questionnaire items, their origin, and reliability and validity (intraclass correlation) ${ }^{37-39}$

\section{Questionnaire item}

1. How many days a week do you drink sugar-sweetened beverages?

2. On a day you drink sugar-sweetened beverages, how many glasses/small bottles $(250 \mathrm{~mL})$, cans $(330 \mathrm{~mL})$ or big bottles $(500 \mathrm{~mL})$ do you drink?

\section{Question derived from}

ENERGY-child questionnaire

Combination ENERGY-child questionnaire and

DOiT questionnaire
Reliability (ICC/k)/validity (ICC/k)

$0.71 / 0.59$

\section{ENERGY}

Glasses/small bottles $(250 \mathrm{~mL})$

$0.59 / 0.24$

Cans (330 mL) 0.53/0.44

Big bottles (500 mL) 0.58/-0.01

DOiT

Cartons/small bottles $(200 \mathrm{~mL})$

$0.74 / 0.12$

Glasses (200 mL) 0.45/0.47

Cans (330 mL) 0.61/0.24

Bottles (500 mL) 0.28/0.17

\section{How many days a week do you drink Added based on Q1 energy drinks or sports drinks?}

\section{On a day you drink energy drinks or} sports drinks, how many small cans/bottles $(250 \mathrm{~mL})$ or big cans/bottles $(500 \mathrm{~mL})$ do you drink?
6. When you eat sweets on a school day, how much sweets do you eat?

7. How many days in the weekend (Saturday/Sunday) do you eat sweets?

\section{How many school days per week do you eat sweets?}

\author{
Added based on Q2
}

DOiT questionnaire (adapted)

Original: 'How many days a week do you eat sweets?'
DOiT questionnaire
$0.71 / 0.21$

DOiT questionnaire (adapted)

Original: 'How many days a week do you eat

$0.66 / 0.60$

sweets?'

8. When you eat sweets on a day in the weekend, how much sweets do you eat?

9. How many school days per week do you
eat snacks?

10. When you eat snacks on a school day, how many small and large snacks do you eat?
DOiT questionnaire
$0.73 / 0.07$

DOiT questionnaire (adapted)

Original: 'How many days a week do you eat snacks?'

$0.50 /-0.11$

DOiT questionnaire

Small snacks $0.62 / 0.13$

Large snacks $0.58 /-0.08$

\section{How many days in the weekend} (Saturday/Sunday) do you eat snacks?

12. When you eat snacks in the weekend (Saturday/Sunday), how many small and large snacks do you eat?
13. How do you usually travel to school? 14. How long does it take you to get from home to school?

\begin{abstract}
DOiT questionnaire (adapted)
Original: 'How many days a week do you eat snacks?'
\end{abstract}

DOiT questionnaire
$0.50 /-0.11$

Small snacks $0.53 / 0.44$

Large snacks $0.64 / 0.08$

DOiT questionnaire

DOiT questionnaire+ENERGY child questionnaire (adapted) does it take you?'
Original: 'If you walk/bike to school, how long
Not in test-retest study

DOiT

Walking 0.65 /zero variance

Biking 0.91/0.68

ENERGY

Walking 0.70/0.59

Biking 0.81/0.66

\section{$0.80 / 0.65$}

\section{What do you usually do when you play} outside at school?

\section{ENERGY-child questionnaire}

ENERGY-child questionnaire-Adapted from Q20
17. I play outside never/1-2 times a week/3- Added 4 times a week/5-6 times a week/every day.

18. When you play outside after school, ENERGY-child questionnaire-Adapted from Q15 what do you do? 
Table 1 Continued

Questionnaire item

Question derived from

Reliability (ICC/k)/validity (ICC/k)

19. When you play outside after school, how Added

long do you play? (fill in the number of hours

per day in table)

20. I like playing sports.

ENERGY-child questionnaire

$0.64 / 0.09$

21. I play sports often/sometimes/never. Added

22a. Do you participate in sports in your free DOiT questionnaire (adapted)

time?

Original:

1. $0.98 / 0.86$

22b. How many times per week do you do this sport?

22c. How many hours per day do you do this sport?

(fill in all sports that you do, the number of times and number of hours per week in the table)

1. 'Do you participate in a sport at a sports club?'

2. $0.94 / 0.78$

2. 'How many hours a week do you do this

3. $0.79 / 0.69$ sport?'

4. $0.76 / 0.96$

5. $0.64 / 0.33$

3. 'Do you participate in a second sport at a

6. $0.64 / 0.45$ sports club?'

4. 'How many hours a week do you do this second sport?'

5. 'Do you participate in sports outside a sports club?'

6. 'How many hours a week do you do these sports?'

23. About how many hours a day do you usually watch television/DVDs/movies on the tablet or iPad in your free time? (fill in the number of hours per day in table)

24. About how many hours a day do you usually play games on your game computer, iPad, smartphone or surfing on the internet in your free time? (fill in the number of hours per day in table, weekdays and weekend days)

\section{ENERGY-child questionnaire (adapted)}

Original: 'About how many hours a day do you

usually watch television in your free time?' (weekdays and weekend days)

ENERGY-child questionnaire (adapted)

Original: 'About how many hours a day do you

usually play games on a computer, or use your

computer in your free time?' (weekdays and

weekend days)
Weekdays $0.67 / 0.63$

Weekend days $0.68 / 0.56$

Weekdays $0.67 / 0.35$

Weekend days $0.67 / 0.65$ interventions. In collaboration with the Action Teams, it is determined how to evaluate the experiences of children with the interventions. The Action Teams can, for example, interview peers or develop a questionnaire. The goal of these evaluations is to see how their peers perceive the interventions and whether quick adaptations need to be made. At the end of each school year, focus groups are organised with children from both the Action Teams and their peers, as well as champions to reflect on the implementation of ongoing interventions and on the empowerment process. Empowerment consists of a combination of individual, organisational and community empowerment. ${ }^{52}$ In our research, we mostly focus on the empowerment of children (individual), but this cannot be evaluated without taking the organisational (school) and community empowerment into account. ${ }^{53}$ The focus groups consist of two exercises. The first exercise is mainly focused on individual empowerment, evaluating what children have learnt about the process of intervention development, how they see their role and qualities. ${ }^{54}{ }^{55}$ The children can choose an intervention idea, which has not been further developed yet. For this intervention, they have to make a timeline with all the steps they need to take from coming up with the idea through to implementation. The researchers guide them through questions; for example, in which order do the steps need to be written down?; do they think they can execute this step by themselves? and if not, do they know where they can get help?. ${ }^{54}$ The second exercise evaluates the organisational and community empowerment. In pairs, the children first indicate which changes happened at school or in the community; then they indicate whether children had any influence on the changes and finally, the findings are discussed in a plenary session. Again the researchers ask questions; for example, how do you feel when you have influence on changes in the community/school?; do you think children have enough influence? and would different changes have been made if children had had more influence? The findings of this focus group provide a critical understanding of the environment, what children have learnt, to what extent children participate in the organisational setting and community, and what collective action has already been taken. ${ }^{53-55}$

Of all hard-copy research data gathered in the PAR meetings, identifiable information is removed and the data are stored in a locked cabinet at the research location until the study is completed. All online data are coded and stored on the VUmc protected drive until 5 years after the completion date of the study; data from the questionnaires, accelerometers, MOPER and personal data are saved with encryption. Hard-copies of the questionnaires and the audio-recordings are also stored at the 
VUmc until 5 years after the study is completed. The three researchers on this project, who are also the authors of this paper, are the only ones who have full access to the trial data. Research assistants have limited and temporary access to copies of the data.

\section{Sample size calculation}

Using a significance level of 0.05 and a power of 0.80 , 180 children per group are needed to detect a difference of $0.15 \mathrm{SD}$ in the primary outcome variables. Taking the dropout and clustering of data within schools into account, we aim to include 240 children per group.

\section{Data analysis}

\section{Effect evaluation}

To test for baseline differences in the dependent variables between control and intervention groups, t-test for continuous variables and $\chi^{2}$ tests for categorical variables are used. Effectiveness of the interventions on dietary behaviour, physical activity, sedentary behaviour, physical fitness and self-rated health is evaluated using multilevel regression analysis with a three-level structure (ie, student, class and school) to adjust for clustering of observations. Analyses are adjusted for age, gender, ethnicity and baseline levels. Data are analysed according to the intentionto-treat principle. All statistical analyses are performed in SPSS, using a significance level of $\mathrm{p}<0.05$.

\section{Process evaluation}

Evaluation of the PAR process and its meetings are mainly performed by the Action Teams themselves. The academic researcher stimulates the children to find patterns and relations in the findings of their own research and assists in interpretation. ${ }^{56}$ Children can, for example, look at the pictures they have taken and write down why they took the picture and what they want to say with the picture. Children can also write down the key issues that come up in the interviews they have conducted and see if they can identify a pattern. By giving children this role in qualitative data analysis, less misinterpretation of data occurs (that would be the case with adults trying to interpret the children's findings).

In addition, all meetings are summarised and include field notes, and key meetings are fully transcribed. ${ }^{49}$ The academic researcher analyses these transcripts to enrich the children's findings. When, for example, the children discuss the pictures they have taken, these discussions may also contain valuable information in addition to the pictures and conclusions of the children. All summaries and transcripts are coded in ATLAS.ti by two researchers to improve the reliability of the study. For the entire process evaluation, an elaborate coding scheme is produced through open coding. ${ }^{56}$ For specific aspects, such as the evaluation of an intervention, coding is done separately resulting in its own coding scheme. For evaluations relating to empowerment, closed coding is used as this will be linked to a conceptual model.

\section{DISCUSSION}

In the Kids in Action project, children are involved throughout the entire research process. This YPAR approach has previously shown promising results for communities in need with respect to researchers' understanding of the community, lowering health disparities, increasing children's skills (eg, research skills and life skills), critical awareness, involvement and empowerment concerning community action. ${ }^{57-59}$

In the Kids in Action project, children are not involved in the first phase of this study, in which partnerships with other stakeholders in the community have to be set up. This is because creating partnerships can be time-consuming and not very interesting for children, and we do not want to lower their spirits. ${ }^{33}$ The partnerships are important in YPAR for creating support in the community for the study ${ }^{5760}$ and are beneficial in the rest of the research process and outcomes.

A difference between this study and most YPAR studies is that children aged 9-12 years are involved as co-researchers, whereas most YPAR studies collaborate with adolescents older than $12 .{ }^{61}$ Younger children can be more easily distracted, have a limited attention span and might need more 'play', all of which should be taken into account when designing the meetings. Meetings should not be too long, should contain fun and playful exercises, and wording should be suitable for the children, while retaining key principles of YPAR. These principles include: sharing power between researchers and children; training children to participate in research and identify needs in their community; teaching children how to become advocates; creating ownership over the process and creating involvement in establishing change in their community. ${ }^{62}$ When all of this is done with care, children between 9 and 12 years old are capable of joining in YPAR research. ${ }^{63-65}$

One implication of working with children aged 9-12 years is that you often have to collaborate intensively with the schools. This could mean that changes in the planning have to be made beforehand or during the project, based on the schools' preferences, holidays and other reasons for cancelling meetings. ${ }^{33}$ Also, the approval and assistance of schools and other community organisations are likely to be needed for implementing the interventions. Because this is a community project, all primary schools in the neighbourhood are included in the intervention and randomisation of schools is not possible. However, the inclusion of comparable control schools is a strength of this study as this is seldom included in PAR. ${ }^{66}$ Another strength of this study is the combination of YPAR with IM, which makes sure that evidence-based strategies are being applied. As far as we know, this has not been done before.

A challenge for all intervention studies in real life is that other initiatives can also take place in the neighbourhood. This is part of usual care and can take place both in the intervention school and the control school neighbourhoods, and may dilute intervention effects. 
Acknowledgements The authors thank the children who participated in the Action Teams for their contribution to this study.

Contributors All the authors worked on the design of this study. MA is the coordinating researcher on the project, coordinating the effect measurements, process evaluation, leading the participatory process and facilitating the Action Teams. TA and MC designed the study. The paper was drafted by MA, with MC and TA providing comments and revisions to drafts. All authors approved the final version.

Funding This work is supported by FNO, grant number 101569. The funder has no role in the study design; collection, analysis and interpretation of data; writing the report and the decision to submit the report for publication.

\section{Competing interests None declared.}

Patient consent for publication Not required.

Ethics approval The Medical EthicsCommittee of the VU University Medical Center approvedthe study protocol (2016.366).

Provenance and peer review Not commissioned; externally peer reviewed.

Open access This is an open access article distributed in accordance with the Creative Commons Attribution Non Commercial (CC BY-NC 4.0) license, which permits others to distribute, remix, adapt, build upon this work non-commercially, and license their derivative works on different terms, provided the original work is properly cited, appropriate credit is given, any changes made indicated, and the use is non-commercial. See: http://creativecommons.org/licenses/by-nc/4.0/.

\section{REFERENCES}

1. CBS. Ervaren gezondheid, zorggebruik en leefstijl bij kinderen tot 12 jaar. 2016 http://statline.cbs.nl/Statweb/publication/?DM=SLNL\&PA= 83716 NED\&D1 $=89 \& D 2=0 \& D 3=\mid \& D 4=0 \& D 5=2 \& V W=T($ Accessed 12 Dec 2017).

2. GGD. Gezondheid in beeld: GGD Amsterdam. 2016 https:// amsterdam.ggdgezondheidinbeeld.nl/jeugd/ (Accessed 12 Dec 2017).

3. Amsterdam G. Staat van gezond gewicht en leefstijl van Amsterdamse kinderen, 2017:1-46.

4. Chi DL, Luu M, Chu F. A scoping review of epidemiologic risk factors for pediatric obesity: Implications for future childhood obesity and dental caries prevention research. J Public Health Dent 2017;77 Suppl 1(Suppl 1):S8-S31.

5. Kumanyika SK, Whitt-Glover MC, Haire-Joshu D. What works for obesity prevention and treatment in black Americans? Research directions. Obes Rev 2014;15 Suppl 4(Suppl 4):204-12.

6. Wang $\mathrm{Y}$, Lim $\mathrm{H}$. The global childhood obesity epidemic and the association between socio-economic status and childhood obesity. Int Rev Psychiatry 2012;24:176-88.

7. Taveras EM, Gillman MW, Kleinman K, et al. Racial/ethnic differences in early-life risk factors for childhood obesity. Pediatrics 2010;125:686-95

8. Wang Y, Beydoun MA. The obesity epidemic in the United Statesgender, age, socioeconomic, racial/ethnic, and geographic characteristics: a systematic review and meta-regression analysis. Epidemiol Rev 2007;29:6-28.

9. CBS. Leefstijl en preventie; geslacht, leeftijd, persoonskenmerken 2016 http://statline.cbs.nl/Statweb/publication/?DM=SLNL\&PA= 83385ned\&D1 $=11-15 \& D 2=a \& D 3=a \& D 4=5-9 \& D 5=0 \& D 6=\mid \& V W=T$ (Accessed 12 Dec 2017).

10. Singh AS, Mulder C, Twisk JW, et al. Tracking of childhood overweight into adulthood: a systematic review of the literature. Obes Rev 2008;9:474-88.

11. Hirasing RA, Fredriks AM, van Buuren S, et al. [Increased prevalence of overweight and obesity in Dutch children, and the detection of overweight and obesity using international criteria and new reference diagrams]. Ned Tijdschr Geneeskd 2001;145:1303-8.

12. Schönbeck $Y$, Talma $H$, van Dommelen $P$, et al. Increase in prevalence of overweight in Dutch children and adolescents: a comparison of nationwide growth studies in 1980, 1997 and 2009. PLoS One 2011;6:e27608.

13. Bahia L, Schaan CW, Sparrenberger K, et al. Overview of metaanalysis on prevention and treatment of childhood obesity. J Pediatr 2018.

14. Wang Y, Cai L, Wu Y, et al. What childhood obesity prevention programmes work? A systematic review and meta-analysis. Obes Rev 2015;16:547-65.

15. Pandita A, Sharma D, Pandita D, et al. Childhood obesity: prevention is better than cure. Diabetes Metab Syndr Obes 2016;9:83-9.
16. Davison KK, Birch LL. Childhood overweight: a contextual model and recommendations for future research. Obes Rev 2001;2:159-71.

17. Metcalf $B$, Henley W, Wilkin T. Effectiveness of intervention on physical activity of children: systematic review and meta-analysis of controlled trials with objectively measured outcomes (EarlyBird 54). BMJ 2012;345:e5888.

18. Cushing CC, Brannon EE, Suorsa KI, et al. Systematic review and meta-analysis of health promotion interventions for children and adolescents using an ecological framework. J Pediatr Psychol 2014;39:949-62.

19. Kamath CC, Vickers KS, Ehrlich A, et al. Clinical review: behavioral interventions to prevent childhood obesity: a systematic review and metaanalyses of randomized trials. J Clin Endocrinol Metab 2008;93:4606-15.

20. Lorenc T, Petticrew M, Welch V, et al. What types of interventions generate inequalities? Evidence from systematic reviews. $J$ Epidemiol Community Health 2013;67:190-3.

21. Chung A, Backholer K, Wong E, et al. Trends in child and adolescent obesity prevalence in economically advanced countries according to socioeconomic position: a systematic review. Obes Rev 2016;17:276-95.

22. Stamatakis E, Wardle J, Cole TJ. Childhood obesity and overweight prevalence trends in England: evidence for growing socioeconomic disparities. Int J Obes 2010;34:41-7.

23. Gittelsohn J, Anderson Steeves E, Mui Y, et al. B'More Healthy Communities for Kids: design of a multi-level intervention for obesity prevention for low-income African American children. BMC Public Health 2014;14:942.

24. Brown HE, Atkin AJ, Panter J, et al. Family-based interventions to increase physical activity in children: a systematic review, metaanalysis and realist synthesis. Obes Rev 2016;17:345-60.

25. Kok G, Peters LWH, Ruiter RAC. Planning theory- and evidencebased behavior change interventions: a conceptual review of the intervention mapping protocol. Psicologia: Reflexão e Crítica 2017;30:19.

26. Bartholomew Eldredge LK, Markham CM, Ruiter RAC, et al. Planning Health Promotion Programs: an Intervention Mapping Approach. 4th ed: John Wiley, 2016.

27. Anselma M, Chinapaw MJM, Altenburg TM. Determinants of child health behaviors in a disadvantaged area from a community perspective: A participatory needs assessment. Int J Environ Res Public Health 2018;15:644.

28. Baum F, MacDougall C, Smith D. Participatory action research. J Epidemiol Community Health 2006;60:854-7.

29. Rodríguez LF, Brown TM. From voice to agency: guiding principles for participatory action research with youth. New Dir Youth Dev 2009;2009:19-34.

30. Ozer EJ. Youth-led participatory action research: Developmental and equity perspectives. Adv Child Dev Behav 2016;50:189-207.

31. Cammarota J, Fine M. Youth Participatory Action Research. In: Cammarota J, Fine M, eds. Revolutionizing education. New York, United States of America: Routledge, 2008:1-11.

32. London JK, Zimmerman K, Erbstein N. Youth-led research and evaluation: Tools for youth, organizational, and community development. New Dir Eval 2003;2003:33-45.

33. Suleiman AB, Soleimanpour S, London J. Youth action for health through youth-led research. J Community Pract 2006;14(1-2):125-45.

34. Municipality of Amsterdam. Bevolking buurten naar herkomstgroepering 2017 https://www.ois.amsterdam.nl/feiten-encijfers/buurten (Accessed 05 Feb 2018).

35. GGD Amsterdam. Gezondheid in beeld. https://amsterdam. ggdgezondheidinbeeld.nl/ (cited 05 Feb 2018).

36. Municipality of Amsterdam. Gebiedsanalyse 2017 noord west stadsdeel noord 2017. https://www.ois.amsterdam.nl/pdf/2017 gebiedsanalyse_17.pdf (Accessed 05 Feb 2018).

37. Singh AS, Vik FN, Chinapaw MJM, et al. Test-retest reliability and construct validity of the ENERGY-child questionnaire on energy balance-related behaviours and their potential determinants: the ENERGY-project. Int J Behav Nutr Phys Act 2011;8:136.

38. Janssen EHC, Singh AS, van Nassau F, et al. Test-retest reliability and construct validity of the DOiT (Dutch Obesity Intervention in Teenagers) questionnaire: measuring energy balancerelated behaviours in Dutch adolescents. Public Health Nutr 2014:17:277-86.

39. Ravens-Sieberer U, Wille N, Badia X, et al. Feasibility, reliability, and validity of the EQ-5D-Y: results from a multinational study. Quality of Life Research 2010;19:887-97.

40. Trost SG, Loprinzi PD, Moore R, et al. Comparison of accelerometer cut points for predicting activity intensity in youth. Med Sci Sports Exerc 2011;43:1360-8. 
41. Fischer C, Yıldırım M, Salmon J, et al. Comparing different accelerometer cut-points for sedentary time in children. Pediatr Exerc Sci 2012;24:220-8.

42. Evenson KR, Catellier DJ, Gill K, et al. Calibration of two objective measures of physical activity for children. J Sports Sci 2008;26:1557-65.

43. Chinapaw MJ, de Niet $M$, Verloigne $M$, et al. From sedentary time to sedentary patterns: accelerometer data reduction decisions in youth. PLoS One 2014;9:e111205.

44. Leyten C, Kemper HCG, Verschuur R. De moper fitheidstest: handleiding en prestatieschalen $9 \mathrm{t} / \mathrm{m} 11$ jarigen. Haarlem: BV Uitgeverij De Vrieseborch, 1982.

45. (Ties) Molenaar HM, Zuidam JM, Selles RW, et al. Age-specific reliability of two grip-strength dynamometers when used by children. $J$ Bone Joint Surg Am 2008;90:1053-9.

46. Hamilton GF, McDonald C, Chenier TC. Measurement of grip strength: validity and reliability of the sphygmomanometer and jamar grip dynamometer. J Orthop Sports Phys Ther 1992;16:215-9.

47. Matsuzaka A, Takahashi Y, Yamazoe M, et al. Validity of the Multistage 20-M Shuttle-Run Test for Japanese Children, Adolescents, and Adults. , 2004:16, 113-25.

48. Nys L, Plowman SA, Looney MA. The reliability and validity of the 20-meter shuttle test in american students 12 to 15 years old. Research Quarterly for Exercise and Sport 1992;63:360-5.

49. Halcomb EJ, Gholizadeh L, DiGiacomo M, et al. Literature review: considerations in undertaking focus group research with culturally and linguistically diverse groups. J Clin Nurs 2007;16:1000-11.

50. Rabiee F. Focus-group interview and data analysis. Proc Nutr Soc 2004;63:655-60.

51. Marshall J, Mead G. Editorial: Self-reflective practice and first-person action research. Action research 2005;3:235-44.

52. Israel BA, Checkoway B, Schulz A, et al. Health education and community empowerment: conceptualizing and measuring perceptions of individual, organizational, and community control. Health Educ Q 1994;21:149-70.

53. Zimmerman M. Empowerment Theory, 2012.
54. Perkins DD, Zimmerman MA. Empowerment theory, research, and application. Am J Community Psychol 1995;23:569-79.

55. Zimmerman MA, Israel BA, Schulz A, et al. Further explorations in empowerment theory: An empirical analysis of psychological empowerment. Am J Community Psychol 1992;20:707-27.

56. Corbin JM, Strauss A. Grounded theory research: Procedures, canons, and evaluative criteria. Qual Sociol 1990;13-3-21.

57. Berg M, Coman E, Schensul JJ. Youth Action Research for Prevention: a multi-level intervention designed to increase efficacy and empowerment among urban youth. Am J Community Psychol 2009;43(3-4):345-59.

58. Foster-Fishman PG, Law KM, Lichty LF, et al. Youth ReACT for socia change: a method for youth participatory action research. Am J Community Psychol 2010;46(1-2):67-83.

59. Checkoway B, Allison T, Montoya C. Youth participation in public policy at the municipal level. Child Youth Serv Rev 2005;27:1149-62.

60. Øen G, Stormark KM. PArticipatory action research in the implementing process of evidence-based intervention to prevent childhood obesity: Project design of the "healthy future" study. $J$ Obes 2013;2013:1-10.

61. Kim J. Youth involvement in participatory action research (par): Challenges and barriers. Critical Social Work 2016;17:38-53.

62. Ozer EJ, Ritterman ML, Wanis MG. Participatory action research (PAR) in middle school: opportunities, constraints, and key processes. Am J Community Psychol 2010;46(1-2):152-66.

63. Caro HE, Altenburg TM, Dedding C, et al. Dutch primary schoolchildren's perspectives of activity-friendly school playgrounds: A participatory study. Int J Environ Res Public Health 2016;13:526.

64. Wang CC. Youth participation in photovoice as a strategy for community change. J Community Pract 2006;14(1-2):147-61.

65. Wilson N, Dasho S, Martin AC, et al. Engaging young adolescents in social action through photovoice. J Early Adolesc 2007;27:241-61.

66. Leykum LK, Pugh JA, Lanham $\mathrm{HJ}$, et al. Implementation research design: integrating participatory action research into randomized controlled trials. Implementation Science 2009;4:69. 